\title{
Blind separation of asymmetric signals based on Synchrosqueezing Wavelet Transform
}

\author{
Peng Chuang, Yang Xiaojing, Zhang Yu \\ School of electronic warfare, National University of Defense Technology
}

\begin{abstract}
A blind separation algorithm based on synchronous squeezing wavelet transform is proposed to solve the blind separation problem of single channel asymmetric signals in satellite communications. First, the algorithm is used to synchronize the strong signal. Then, the signal time-frequency curve is extracted by synchronous extrusion wavelet transform. Finally, the weak signal interference is filtered out from the mixed signal except the noise and the main frequency of the strong signal. Therefore, the ber of the strong signal demodulation is reduced. The algorithm has the characteristics of blind separation of single channel asymmetric signals without prior information sequence sent by cooperative communicators. The simulation results show that the performance of strong signal demodulation error is better than that of mixed signal direct hard decision.
\end{abstract}

\section{Introduction}

Single channel mixed signal is a hotspot in the field of signal processing ${ }^{[1]}$. In cooperative communication, because both sides have prior information, the known sequence and mixed sequence can be used to cancel, and the mixed signal can be separated simply to get the required information sequence. However, in noncooperative communication, there is no prior information, and the method of cooperative communication cannot be used to obtain useful signals ${ }^{[2]}$. At the same time, because the mixed signal is composed of fixed mode aliasing, it can not be converted from under-determined signal mode to fixed signal mode by receiving signals from multi-terminal antennas $^{[3]}$.

Blind separation of asymmetric signals has been a hot topic in the research of single channel blind source separation. At present, some achievements have been made. Based on the idea of serial interference cancellation in multi-user detection technology, a blind separation algorithm for asymmetric signals with iterative reconstruction cancellation processing is proposed. However, the algorithm is complex and needs multiple reconstruction to reduce the bit error rate[4]. Based on the predictability of weak signal waveform, a nonlinear filter is introduced to predict the weak signal waveform, and the predicted value is subtracted from the mixed signal to reduce the demodulation error rate of the strong signal[5]. Blind separation algorithm based on soft information joint correction improves the demodulation performance of strong and weak signals, but the algorithm judges the wrong signal according to the constellation diagram. When the signal frequency offset is large, the constellation offset and the algorithm effect is reduced[6]. Q filter algorithm is pengchuang94@126.com used to extract the real information under strong random error, but this algorithm is not suitable for frequency band aliasing signal[7]. An algorithm using wavelet transform to extract real information from complex environment is porposed in [8]. The problem of weak signal detection under strong signal coverage is studied in [9]. In this paper, the hard decision value of the strong signal is subtracted from the mixed signal processing, and the residual signal is detected by energy detection to achieve weak signal detection under strong signal coverage, but the performance of strong signal demodulation error is required to be high.

A blind source separation algorithm based on synchronous extrusion wavelet transform is proposed. The algorithm has the characteristics of blind separation of single channel asymmetric signals without prior information sequence sent by cooperative communicators. The simulation results show that the performance of strong signal demodulation error is better than that of mixed signal direct hard decision.

\section{Asymmetric signal model and description of blind separation problem}

In this paper, the single channel mixed signal neutron signal adopts QPSK modulation mode, and the signal model is:

$$
z(t)=y_{1}+y_{2}+n
$$

The $y_{1} 、 y_{2}$ in the upper form can represent strong and weak component signals respectively. The $\mathrm{n}$ is a stationary Gauss white noise with a mean value of 0 and a variance of $\sigma_{v}^{2}$. 
The above formula can be further expressed as:

$$
\begin{aligned}
& y_{1}=h_{1} e^{j\left(2 \pi f_{1} t+\phi_{1}\right)} \sum_{n=-\infty}^{\infty} a_{n} g_{1}\left(t-n T-\tau_{1}\right) \\
& y_{2}=h_{2} e^{j\left(2 \pi f_{2} t+\phi_{2}\right)} \sum_{n=-\infty}^{\infty} b_{n} g_{2}\left(t-n T-\tau_{2}\right)
\end{aligned}
$$

Blind separation of single channel asymmetric signals is to recover strong signal $y_{1}$ and weak signal $y_{2}$ when mixed signals $z(t)$ are received with little or no prior information. There are three steps to solve this problem. The first step is to synchronize the asymmetric mixed signal $z(t)$ by strong signal and get the mixed signal sequence $z(k)$. In the second step, synchronous extrusion wavelet transform is applied to the signal sequence $z(k)$ to get the mixed signal time-frequency curve, and the timefrequency curve is processed to remove the noise outside the main frequency of the signal and the interference of the weak signal frequency. In the third step, synchronous extrusion wavelet inverse transform is used to get the processed sequence $y(k)$, hard decision is made on $y(k)$, and the strong signal decision value is obtained by demodulation. The weak signal information is obtained by subtracting the strong signal from the mixed signal and demodulating the weak signal $y_{2}(t)$.

\section{Blind separation algorithm for asymmetric signals}

\section{1 low complexity blind separation structure for asymmetric signals}

Since the timing information of strong signal and weak signal is different, synchronization of received mixed signals should first be synchronized. According to the method of single signal demodulation ${ }^{[9]}$, matched filtering, timing synchronization and carrier synchronization are applied to the strong signal. This is equivalent to sampling the mixed signal according to the symbol rate of the strong signal. For weak signals, a sampling rate transformation is performed. And the frequency offset, phase and time delay of the weak signal will change. In order to make the derivation process more applicable, if the strong signal sequence is $\mathrm{R}$ and the weak signals are $\mathrm{T}$, then the signal model after synchronization is established.

$$
\begin{aligned}
z(k)= & A_{s} a_{s}(k)+\sum_{i=1}^{N} s_{i}\left(k T_{s}\right)+n\left(k T_{s}\right) \\
s_{i}\left(k T_{s}\right)= & A_{i} \exp \left(\mathrm{j} 2 \pi f_{s i} k T_{s}+\varphi_{s i}\right) \\
& \sum_{m=-\infty}^{\infty} a_{i}(m) g_{i}\left(k T_{s}-m T_{i}-\tau_{s i}\right)
\end{aligned}
$$

$\tau_{s i} 、 \varphi_{s i} 、 f_{s i}$ are new delay, phase and frequency offset of weak signal after synchronization processing. Strong signals make hard decisions, and mixed signals subtract strong signals from hard decision results.

$$
\begin{aligned}
z^{\prime}(k) & =\left(A_{s} a_{s}(k)-A_{s}^{\prime} a_{s}^{\prime}(k)\right) \\
& +\sum_{i=1}^{N} s_{i}\left(k T_{s}\right)+n\left(k T_{s}\right)
\end{aligned}
$$

$z^{\prime}(k)$ is composed of strong signal decision error, weak signal and noise. $a_{s}^{\prime}(k)$ is the hard decision value of strong signal and the signal amplitude estimation value is $A_{s}^{\prime}$.

Based on this signal model, each weak signal in the center can be demodulated separately to realize blind separation of asymmetric signals $z^{\prime}(k)$, and the block diagram is shown in Figure 1.

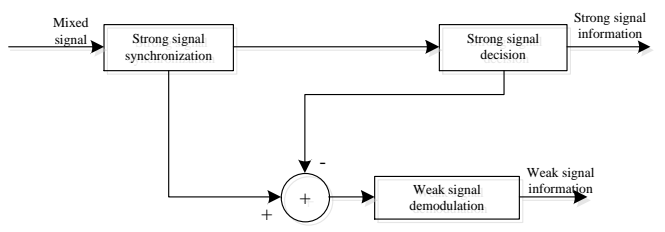

Figure 1. Blind separation structure with low complexity

\subsection{Reducing the bit error rate of strong signal by Synchrosqueezing Wavelet Transform}

In general, weak signal and noise will affect the strong signal demodulation and can be regarded as a part of the noise. That is:

$$
n_{\text {new }}=\sum_{i=1}^{N} s_{i}\left(k T_{s}\right)+n\left(k T_{s}\right)
$$

At this point, the new noise $n_{\text {new }}$ composed of weak signal and original noise is no longer Gaussian white noise, and when the number of weak signals is large and the power is strong, the noise will interfere with the strong signal. Synchronous extrusion wavelet transform is proposed by Daubechies ${ }^{[10]}$ et al. The algorithm is applied to seismic wave signal processing in [11][12]. In this paper, there are obvious frequency differences between strong and weak signals or between signal and noise. Inspired by this paper, we establishes a blind separation model for asymmetric signals and applies SWT to the field of satellite signal processing, which effectively improves the performance of the blind separation algorithm. The Blind Separation Model is based on wavelet transform. The wavelet coefficients are reconstructed, and the timefrequency curves are extracted by combining wavelet transform and energy redistribution.

The mixed signal sequence is SWT and arranged in frequency domain according to the energy. At this time, the strong signal is obviously different from the weak signal. On this basis, after filtering out the weak signal and noise disturbance besides the main frequency of the mixed signal, the wavelet inverse transform of synchronous extrusion is carried out. Hard decision is made on the processed signal to reduce the error rate of strong signal demodulation and improve the performance of the whole blind separation structure. 
To facilitate theoretical analysis, the mixed signal $s(t)$ model is simplified, which includes a strong signal and a $N$ weak signal:

$$
s(t)=\sum_{i=1}^{N+1} f_{i}(t)=\sum_{i=1}^{N+1} A_{i} \cos \left[2 \pi \varphi_{i}(t)\right]
$$

$A_{i}>0, \varphi_{i}^{\prime}(t)>0$ 。SWT can accurately analyze and extract the frequency $f_{i}$ of each component in $s(t)$.

If the $f_{i}(t)=A_{i} \cos \left[2 \pi \varphi_{i}(t)\right]$ in the signal satisfies the conditions as below:

$$
\begin{array}{r}
A_{i} \in C^{1}(\mathbf{R}) \bigcap L^{\infty}(\mathbf{R}), \varphi_{i} \in C^{2}(\mathbf{R}) \\
\inf _{t \in \mathbf{R}} \varphi_{i}{ }^{\prime}(t)>0 \\
\left|A_{i}{ }^{\prime}(t)\right|,\left|\varphi_{i}{ }^{\prime \prime}(t)\right| \leq \gamma\left|\varphi_{i}{ }^{\prime}(t)\right|
\end{array}
$$

The signal $f_{i}(t)$ is called IMT (intrinsic-mode-type), sign it as $A_{\gamma, d}$.

$A_{\gamma, d}$ consists of several oscillating components. The amplitude of the component changes slowly and the frequency is smooth enough. When the difference between high-frequency component and low-frequency component is obvious, SWT can be completely separated according to the difference of time-frequency component.

(1) The continuous wavelet transform of mixed signals $s(t) \in A_{\gamma, d}$ is expressed as follows:

$$
W_{s}(a, b)=\int s(t) a^{-1 / 2} \psi^{*}\left(\frac{t-b}{a}\right) \mathrm{d} t
$$

$a$ is the scale factor, $b$ is the time factor and $\psi^{*}$ is the wavelet generating function. When the continuous wavelet transform is transformed to the frequency domain, the expression is:

$$
W_{s}(a, b)=\frac{1}{2 \pi} \int a^{-1 / 2} s(\xi) \psi^{*}(a \xi) \exp (\mathrm{j} b \xi) \mathrm{d} \xi
$$

According to formula (12), the wavelet coefficients $W_{s}(a, b)$ will be centrally distributed near the scale domain $a=W_{0} / W$.

(2) The instantaneous frequency can be estimated preliminarily by derivation of wavelet coefficients.

$\omega_{\mathrm{s}}(\mathrm{a}, \mathrm{b})= \begin{cases}-\mathrm{j} \mathrm{W}_{\mathrm{s}}(\mathrm{a}, \mathrm{b})^{-1} \frac{\partial}{\partial \mathrm{b}} \mathrm{W}_{\mathrm{s}}(\mathrm{a}, \mathrm{b}),\left|\mathrm{W}_{\mathrm{s}}(\mathrm{a}, \mathrm{b})\right|>0 \\ \infty, & \left|\mathrm{W}_{\mathrm{s}}(\mathrm{a}, \mathrm{b})\right|=0\end{cases}$

According to the instantaneous frequency estimation obtained by formula (13), the mapping relation of $(a, b) \rightarrow\left(\omega_{s}(a, b), b\right)$ can be obtained, and the transformation of wavelet coefficients from "time-scale domain" to "time-frequency domain" can be realized.

(3) The wavelet coefficient $W_{s}(a, b)$ is extruded:

$$
\begin{aligned}
T_{s, \gamma}^{\delta}(b, \omega)= & \int_{A_{\gamma, s(b)}} W_{s}(a, b) \\
& \times \frac{1}{\delta} h\left(\frac{\omega-\omega_{s}(a, b)}{\delta}\right) a^{-3 / 2} \mathrm{~d} a
\end{aligned}
$$

The threshold is $\gamma^{*}=\gamma^{1 / 3}$ and the accuracy is $\delta$. In addition ${ }^{[11]}$ :

$$
\begin{gathered}
\left|\omega_{f}(a, b)-\varphi^{\prime}{ }_{k}(b)\right|<\gamma^{*} \\
\left|\lim _{\delta \rightarrow 0}\left(\frac{1}{C_{\psi}} \int_{\left\{|| \omega_{s}(a, b)-\varphi_{k}^{\prime}(b) \mid<\gamma^{*}\right\}} T_{s, \gamma^{*}}^{\delta}(b, \omega) \mathrm{d} \omega\right)-A_{i}\right| \leq \gamma^{*}
\end{gathered}
$$

The formula (15) shows the instantaneous frequency distribution near the instantaneous frequency of the original signal component after the SWT transformation. The formula (16) shows that each component $f_{i}$ can be reconstructed by inverse transform of continuous wavelet transform, and amplitude $A_{i}$ can be recovered by absolute value.

The inverse transformation of Synchrosqueezing Wavelet Transform is as follows ${ }^{[13]}$ :

$$
s(t)=\operatorname{Re}\left[C_{\psi}^{-1} \sum_{i} W_{s}(a, b) a_{i}^{-2 / 3}(\Delta a)_{i}\right]
$$

$\varphi^{*}(\xi)$ is the Fourier transform of wavelet conjugation, Re means the real part, $a_{i}$ is the discrete scale, and $i$ is the scale number. The above is the main process and theory of synchronous extrusion wavelet transform.

The carrier frequency of the first sub-signal is $150 \mathrm{kHz}$, and the carrier frequency of the second subsignal is $100 \mathrm{kHz}$. The distribution of Single-channel mixed signal after SWT in frequency domain is shown in Figure 2 below.

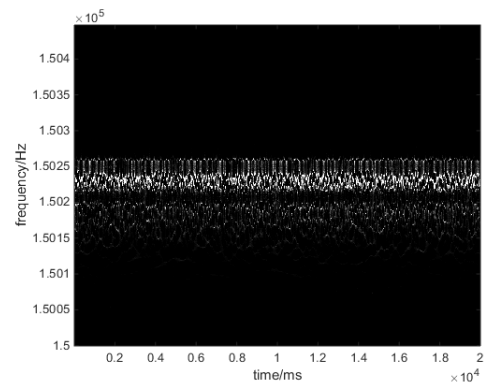

Figure 2. Frequency domain diagram

As can be seen from Fig. 1, the energy of the two subsignals is the largest, mainly distributed in the vicinity of their respective carrier frequencies, and the color is brighter. At this point, the whole APCMA signal blind separation structure block diagram is shown in Figure 3 below.

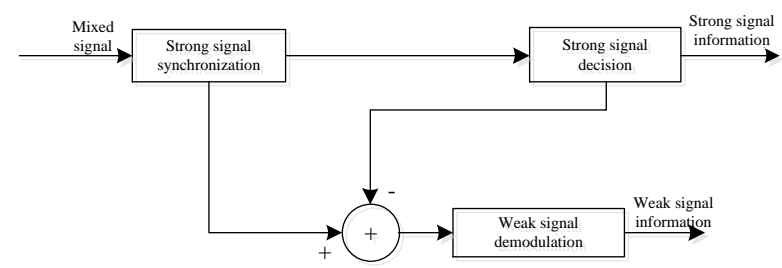

Figure 3. Blind separation structure of APCMA signal

At present, the reconstruction cancellation algorithm is often used to achieve blind separation of APCMA signals. This algorithm uses iterative reconstruction cancellation to reduce the bit error rate of strong signal demodulation. The algorithm needs to reconstruct the weak signal based on the demodulation of the strong and weak signals. The implementation of the algorithm is more complex and 
requires many iterations. At the same time, the algorithm is susceptible to noise, strong signal demodulation error, signal reconstruction error and reconstruction error is difficult to cancel.

\section{Simulation experiment}

In order to verify the performance of the algorithm, this paper explores the influence of mixed signal SNR and mixed signal amplitude ratio on the BER performance of signal demodulation through the following two experiments. In the simulation experiment, the error rate of signal demodulation after separation reflects the performance of the algorithm. The carrier frequency of the first signal is $f_{1}=100 \mathrm{khz}$ and the carrier frequency of the second signal is $f_{2}=200 \mathrm{khz}$.

Experiment 1 Studied the influence of mixed signal to noise ratio on the performance of the algorithm.

To study the effect of signal-to-noise ratio (SNR) on the performance of the algorithm, the amplitude ratio of two sub-signals is fixed to $1: 0.5$, and the SNR of the mixed signal is changed. The performance of the algorithm is affected by SNR as shown in Fig 4.

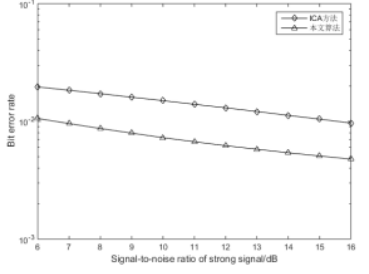

(a)

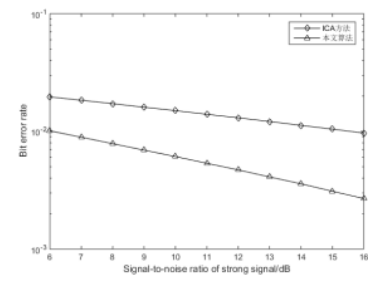

(b)
Figure 4. Signal demodulation bit error rate

In Fig 4,(a) is strong signal demodulation bit error rate and (b) is weak signal demodulation bit error rate (b) From Fig 4, we can see that the proposed algorithm has better performance than ICA in demodulation error rate, which decreases with the increase of signal-to-noise ratio of mixed signals. This is because the interference of noise decreases with the increase of signal to noise ratio.

Experiment 2 Studied the influence of mixed signal amplitude ratio algorithm on the performance.

To study the effect of amplitude ratio of mixed signal on the performance of the algorithm, the signal-to-noise ratio of mixed signal is fixed to $15 d B$, and the amplitude of weak signal is changed to $[0.4 \sim 0.8]$. The performance of the algorithm is affected by the amplitude ratio of mixed signal as shown in Figure 5.

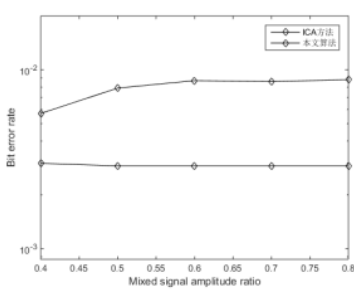

(a)

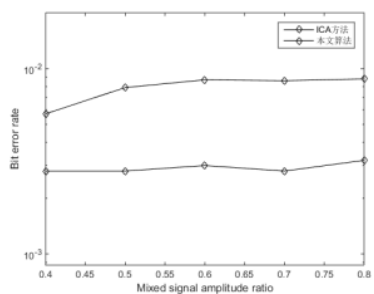

(b)
Figure 5. Signal demodulation bit error rate
As shown in Fig. 5, the performance of the algorithm varies with the amplitude ratio of the mixed signal. The algorithm is less affected by the amplitude ratio and almost unchanged.

A blind separation algorithm based on synchronous extrusion wavelet transform is proposed for single channel asymmetric mixed signals in satellite communications. The algorithm has the characteristics of blind separation of single channel asymmetric signals without prior information sequence sent by cooperative communicators. The simulation results show that the performance of strong signal demodulation error is better than that of mixed signal direct hard decision.

\section{References}

1. Wang G Single channel high order MPSK signal separation method [D]. U S China, 2017.

2. Guo Y .High-efficiency time delay estimation method for single channel co-frequency mixed signals $[\mathrm{J}] . \mathrm{S} E$ 2014.

3. Guo Y. Key technologies for blind demodulation of PCMA signals [D]. The PLA Information Engineering University, 2015.

4. Tu S L, Zheng H, Gu N. Sigle-channel blind separation of two QPSK signals using per- survivor processing [C]//IEEE Asia Pacific Conference 2008

5. Yang Y. Single channel blind separation of the same frequency mixed signals using Gibbs sampling. $[\mathrm{J}] \mathrm{J}$ C, 2015

6. Huang Q, Asymmetric PCMA blind separation algorithm based on joint soft information correction [J]. J C, 2017.

7. Hu Shao-lin. High fidelity error tolerant Q- filtering algorithm for dynamic measurement data[J]. J A 2016

8. Huang Q. Blind separation of asymmetric PCMA signals based on nonlinear filtering [J]. J S, 2017.

9. Liao C, A new detection algorithm for detecting weak signals from strong signal masking [J].J S, 2010

10. Daubechies I, Lu J, Wu H T. Synchrosqueezed wavelet transforms: a tool for empirical mode decomposition[J]. M 2009.

11. Anvari R, Siahsar M A N, Gholtashi S, et al. Seismic random noise attenuation using synchrosqueezed wavelet transform and low-rank signal matrix approximation[J]. IEEE Transactions on Geoscience \& Remote Sensing, 2017

12. Mousavi S M, Langston C A, Horton S P. Automatic microseismic denoising and onset detection using the synchrosqueezed continuous wavelet transform $[\mathrm{J}]$. Geophysics, 2016,

13. Daubechies I, Lu J, Wu H T. Synchrosqueezed wavelet transforms: an empirical mode decomposition-like tool[J]. Applied \& Computational Harmonic Analysis, 2011, 30(2):243-261. 